\title{
Surface Plasmon Resonance in Periodic Hexagonal Lattice Arrays of Silver Nanodisks
}

\author{
Jinlian Hu, ${ }^{1}$ Cong Wang, ${ }^{1}$ Shikuan Yang, ${ }^{2}$ Fei Zhou, ${ }^{2}$ Zhigang Li, ${ }^{2}$ and Caixia Kan ${ }^{3}$ \\ ${ }^{1}$ School of Materials Science and Engineering, and Anhui Key Laboratory of Metal Materials and Processing, \\ Anhui University of Technology, Ma-An-Shan, Anhui 243002, China \\ ${ }^{2}$ Key Laboratory of Materials Physics, Anhui Key Laboratory of Nanomaterials and Nanotechnology, Institute of Solid State Physics, \\ Chinese Academy of Sciences, Hefei, Anhui 230031, China \\ ${ }^{3}$ College of Science, Nanjing University of Aeronautics and Astronautics, Nanjing 210016, China
}

Correspondence should be addressed to Jinlian Hu; jlhu72@163.com and Shikuan Yang; skyang@issp.ac.cn

Received 30 November 2012; Accepted 18 January 2013

Academic Editor: Yue Li

Copyright (C) 2013 Jinlian Hu et al. This is an open access article distributed under the Creative Commons Attribution License, which permits unrestricted use, distribution, and reproduction in any medium, provided the original work is properly cited.

\begin{abstract}
The surface plasmon resonance (SPR) of periodic hexagonal lattice arrays of silver nano-disks positioned on glass slides is studied using finite-difference time domain (FDTD) simulations. We investigate numerically the influence of diameter of nano-disks and the gap between nano-disks on SPR transmission spectra and electric field enhancement. We find a strong dependence of resonance wavelength on diameter of the nanodisks. With increasing the gap, electric field enhancement factor could significantly increase and reach a maximum value, which indicates that a special long-range interaction plays an important role. This study is useful for optical modulation applied in near or far field optics, sensing and data storage, and solar cell.
\end{abstract}

\section{Introduction}

Surface plasmon resonance (SPR) can be generated in metal nanostructures such as gold, silver, and copper, which are promising materials in the fields of optoelectronics and plasmonics [1-5]. The SPR in periodic arrays has aroused great interest because of its uniform optical properties which can be applied in integrated optoelectronics device [610]. Previously, a silver nanostructure in periodic square lattice for label free nano-biosensors was proposed in [6] and SPR in periodic square lattice of gold nanodisks for broadband light harvesting was investigated in [7]. Recently, Yang et al. [11] developed surface pattern fabrications via a dewetting process on the surface of a bowl template derived from a monolayer colloidal crystal template composed of polystyrene (PS) spheres in periodic hexagonal lattice arrays, and each bowl in the template can be treated as a separate reactor, resulting in uniformly structured surface patterns, and then the periodic hexagonal lattice arrays of metal nanodisks can be easily formed. However, the theoretical simulation about the SPR properties of periodic hexagonal lattice arrays of silver nanodisks has scarcely been reported.

In this study, we show how by tuning the diameter and the gap to control the transmission and E-field enhancement properties of periodic hexagonal lattice arrays of silver nanodisks through the interactions of nanodisks. The longrange interaction of nano-particles is observed when $E$ field enhancement reaches a maximum value at the critical gap. The optical properties (transmission spectra and field enhancement) of silver nanodisk arrays are systematically studied numerically using Finite Difference Time Domain method.

\section{Sample and Methods}

Figure 1 shows the schematic representation of periodic hexagonal lattice arrays of $\mathrm{Ag}$ nanodisks on $\mathrm{SiO}_{2}$ glass substrate. The height of nanodisk is held constant at $20 \mathrm{~nm}$. 


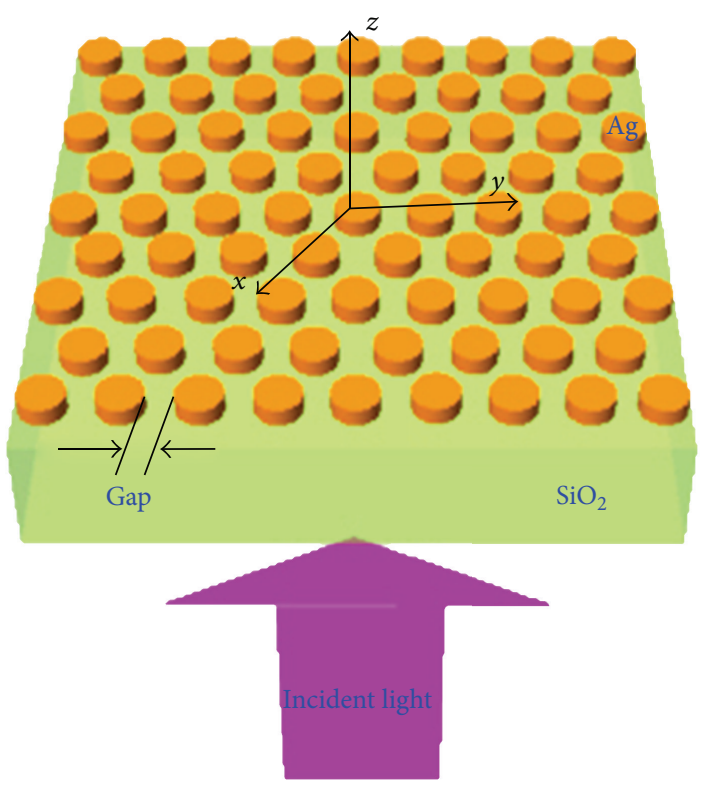

FIGURE 1: The schematic representation of periodic hexagonal lattice arrays of silver nanodisks positioned on glass slides.

The transmission spectra and E-field enhancement for the hexagonal array was calculated using a finite-difference time domain method (FDTD Solutions, from Lumerical). The calculations are set up as a three-dimensional system with a 2-nm resolution grid, for $500 \mathrm{fs}$. A plane wave source is chosen at a working wavelength range of 300-700 nm. The arrays are illuminated by a $x$-polarized plane wave source shining from the glass side towards the positive $z$ direction, which is perpendicular to the plane of the platform. Perfectly matched layers (PMLs) are used on the $z$-axis boundaries, while periodic boundaries conditions (PBCs) are placed on the $x$ - and $y$-axis ones. The transmitted power is collected at positive $z$-direction at ends of the domain. The dielectric constant of the glass (silicon dioxide) and silver are described by the Palik values [12], which were provided in the material database from the software. The calculation of electric field enhancement factor, the relative total electric field intensity, and its image plot are obtained from $|E|^{2} /\left|E_{0}\right|^{2}$, and they are calculated on the top surface of nanodisks.

\section{Results and Discussion}

For comparison, Figures 2(a), 2(b), and 2(c) show relative field intensities $|E|^{2} /\left|E_{0}\right|^{2}$ distribution on the top surface of nanodisks along $x y$ plane at respective resonance wavelength for three different patterns (i.e., single nanodisk, seven nanodisks system, and periodic hexagonal arrays). The transmission spectra of the above three patterns are presented in Figure 2(d). In the Figure 2, the diameter and the gap are $100 \mathrm{~nm}$ and $70 \mathrm{~nm}$, respectively. It can been seen that the periodic hexagonal array has a strong SPR transmission peak at $555 \mathrm{~nm}$, which is slightly different from the peak of seven nanodisks system at $564 \mathrm{~nm}$, and much more different from the one of the single nanodisk at $600 \mathrm{~nm}$. As for the $E$-field enhancement factor at respective resonance wavelength, the maximum value $\left|E_{\max }\right|^{2} /\left|E_{0}\right|^{2}$ of periodic hexagonal array is about 180 , which is also slightly different from the maximum value of seven nanodisks system of 350, and much more different from the single nanodisk of 700 . These results indicate that the interaction between nanodisks cannot be ignored. In the following, we present the influence of nanodisks diameter and gap on SPR transmission spectra and $E$-field enhancement considering electromagnetic interaction.

3.1. The Influence of Diameter of Nanodisks on Transmission Spectra and E-Field Enhancement. The SPR transmission spectra of the periodic structures have been calculated for diameter between 20 and $300 \mathrm{~nm}$ with fixed gap of $70 \mathrm{~nm}$, as shown in Figure 3(a). As we can see, the SPR peak significantly red shifts from $414 \mathrm{~nm}$ to $633 \mathrm{~nm}$ with great change about $221 \mathrm{~nm}$ when the particle diameter increases from $20 \mathrm{~nm}$ to $150 \mathrm{~nm}$. The full width at half maximum (FWHM) and intensity of the SPR peak also greatly increase with increasing diameter.

In Mie theory, extinction is directly proportional with the density of the nanostructures [1]. Obviously, the increase in diameter keeping the gap constant is expected to increase a real density of $\mathrm{Ag}$ in the periodic hexagonal arrays, which leads to the increase in the amplitude of resonance intensity. With increasing the size of each particle, the electromagnetic wave incident on the particles should no longer be considered to have a negligible phase difference across the single particle dimension, and the dipole resonance peak starts to shift towards longer wavelengths and 


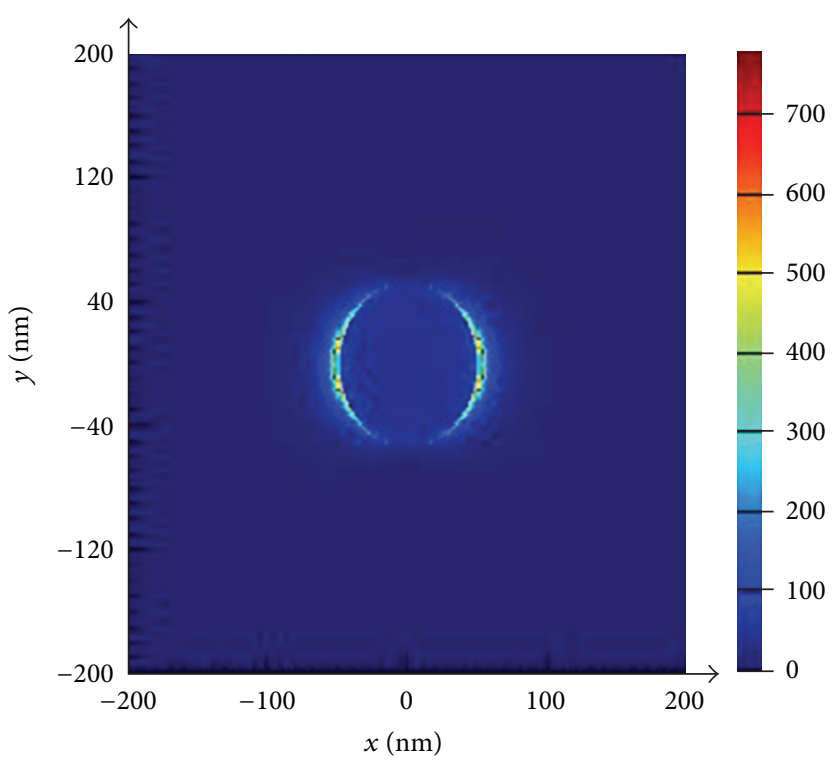

(a)

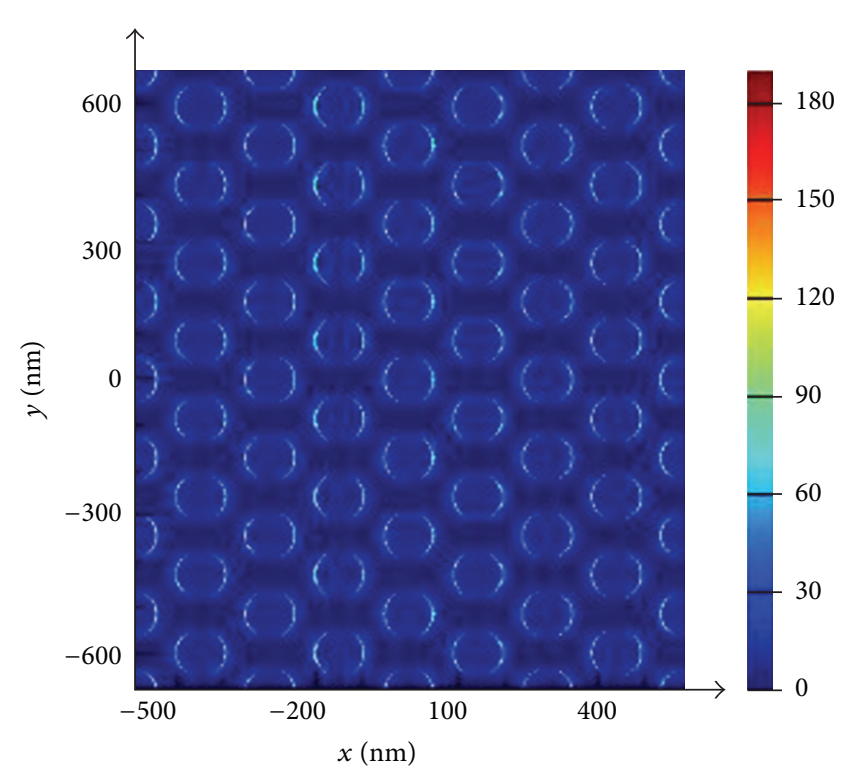

(c)

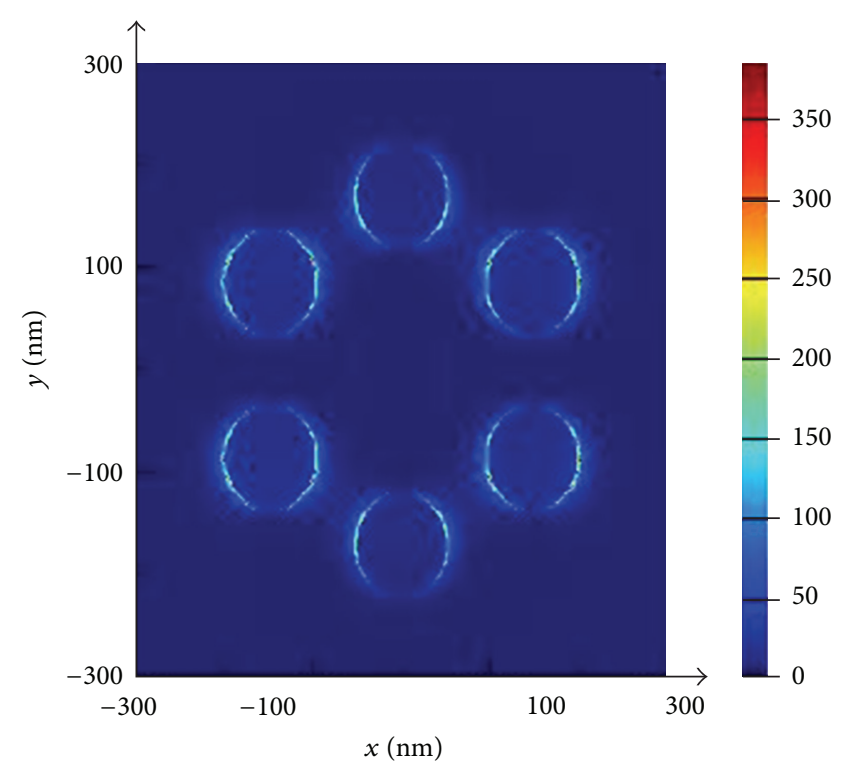

(b)

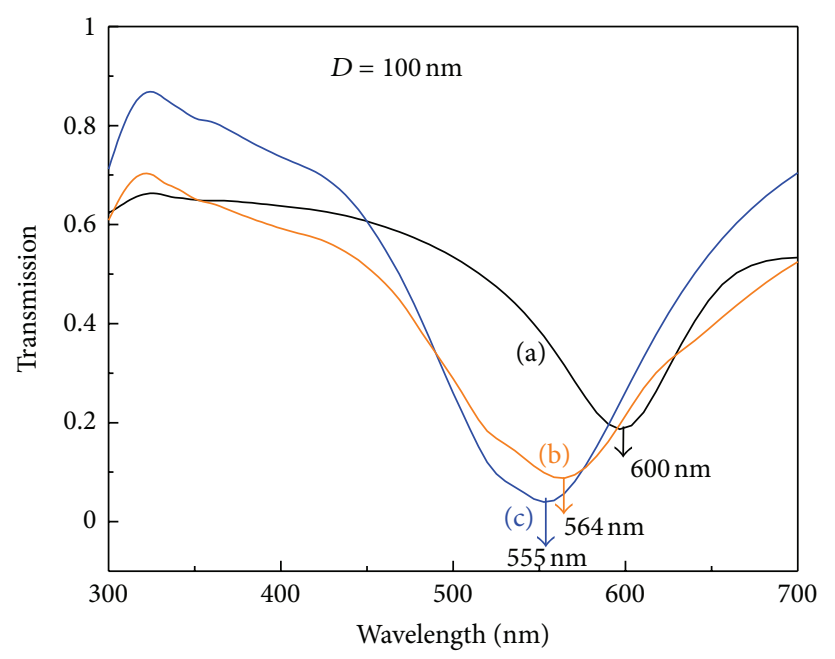

(a) single nanodisk

(b) seven nanodisks, gap $=70 \mathrm{~nm}$

(c) periodic array, gap $=70 \mathrm{~nm}$

(d)

FIGURE 2: The relative field intensities $\left(|E|^{2} /\left|E_{0}\right|^{2}\right)$ distribution on the top surface of nanodisks along $x y$ plane at respective resonance wavelength for three different patterns: (a) single nanodisk, (b) seven nanodisks system, and (c) periodic hexagonal arrays. (d) The transmission spectra of the above three patterns. The diameter and the gap are $100 \mathrm{~nm}$ and $70 \mathrm{~nm}$, respectively.

SPR peak gets wider due to phase retardation $[1,2]$. Here, phase retardation plays a dominant role in leading to the significant change in peak position and FWHM When the diameter of each nanodisk changes. In addition to phase retardation, maybe the near field coupling between nanodisks gets stronger, which leads to broader peak when the diameter increase.
Figure 3(b) shows E-field enhancement factor of periodic arrays with fixed gap of $70 \mathrm{~nm}$ and different diameters illuminated at three laser wavelengths $(488 \mathrm{~nm}, 532 \mathrm{~nm}$, $632.8 \mathrm{~nm}$ ). It can be seen that irradiation by the laser light with a wavelength of $532 \mathrm{~nm}$ or 632.8 has better $E$-field enhancement effect than by the laser light with a wavelength of $488 \mathrm{~nm}$ when the diameter increases up to $100 \mathrm{~nm}$ because 


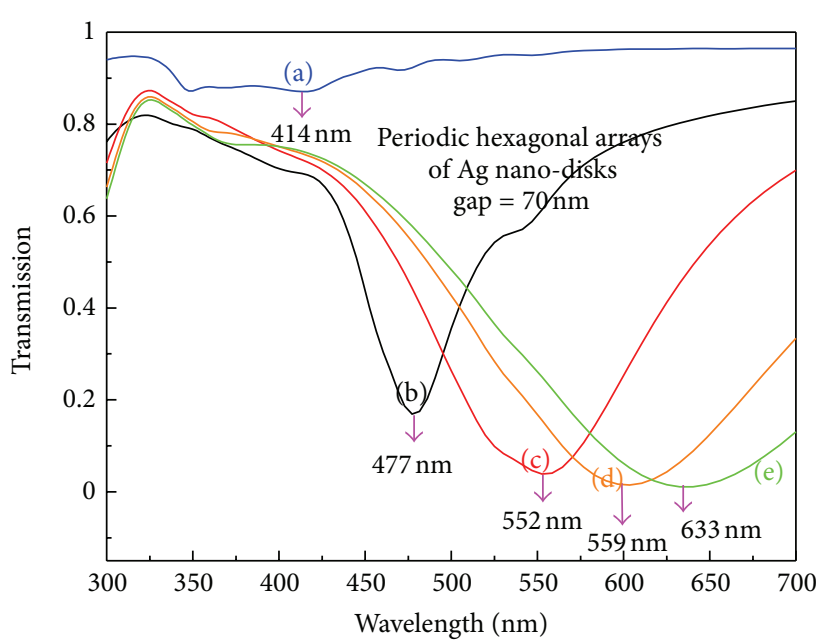
(a) $D=20 \mathrm{~nm}$
(b) $D=60 \mathrm{~nm}$
(c) $D=100 \mathrm{~nm}$

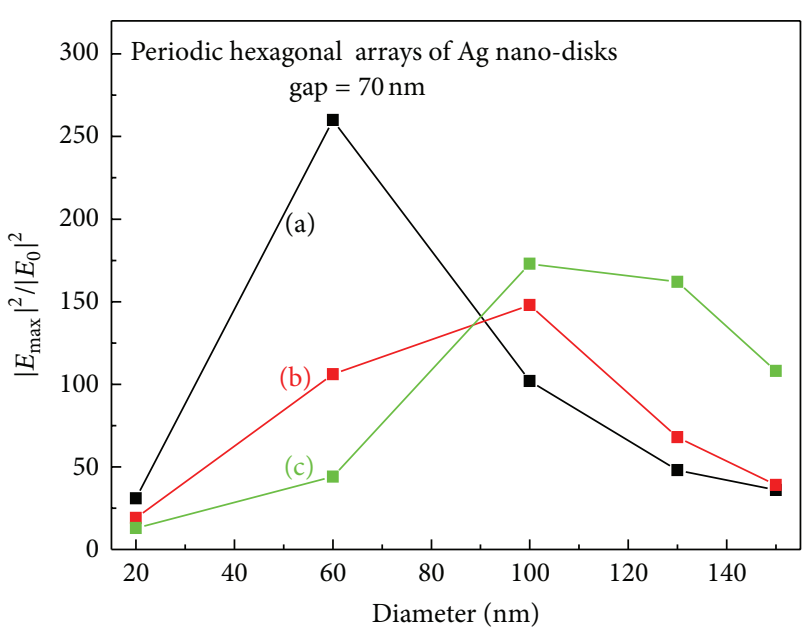

(a) $\lambda=488 \mathrm{~nm}$

(b) $\lambda=532 \mathrm{~nm}$

(c) $\lambda=632.8 \mathrm{~nm}$ (a)

(d) $D=130 \mathrm{~nm}$
(e) $D=150 \mathrm{~nm}$ (b)

Figure 3: (a) The transmission spectra of periodic arrays with different diameters $D$ between $20 \mathrm{~nm}$ and $150 \mathrm{~nm}$. (b) $E$-field enhancement factor of periodic arrays with different diameters illuminated at three laser light wavelengths ( $488 \mathrm{~nm}, 532 \mathrm{~nm}, 632.8 \mathrm{~nm})$. The gap between the nanodisks is fixed at $70 \mathrm{~nm}$.

the resonance wavelength red shifts with the increase in diameter.

\subsection{The Influence of the Gap between Nanodisks on Transmis-} sion Spectra and E-Field Enhancement. The SPR transmission spectra of the periodic structures have been calculated for different gaps with fixed diameter of $100 \mathrm{~nm}$, as shown in Figure 4(a). With increasing gap, the intensity of the SPR extinction peak decreases as the density of silver nanodisks decreases and the SPR peak position has slight change. However, the FWHM of the peak gets much narrower with the increase in the gap, which could indicate the near-field coupling becomes weak. The strength of nearfield coupling could be estimated from the FWHM of SPR peak.

In principle, two types of interaction can be distinguished: near-field coupling and far-field (dipolar) interaction. Near-field coupling is relevant for nearly touching particles due to the short range of the electromagnetic near fields in the order of some tens of $\mathrm{nm}$. On the other hand, farfield interaction is mediated by the nanostructures' scattered light fields [13]. The near-field coupling of silver nanoparticles pairs is efficient at few tens nanometers distance and gradually vanishes when the $(D+$ gap $) / D>2.5$ and long range dipole-dipole interaction is stronger as the gap increases in the periodic pattern [14].

Figure 4(b) shows $E$-field enhancement factor on the top of nanodisks along $x y$ plane for periodic hexagonal arrays with fixed diameter of $100 \mathrm{~nm}$ and different gaps illuminated at three laser wavelengths ( $488 \mathrm{~nm}, 532 \mathrm{~nm}, 632.8 \mathrm{~nm}$ ), which suggests the irradiation by the laser light with a wavelength of $532 \mathrm{~nm}$ or $632.8 \mathrm{~nm}$ has better $E$-field enhancement effect than by laser light with a wavelength of $488 \mathrm{~nm}$ under such condition.

Figure 4(c) presents $E$-field enhancement factor on the top surface of nanodisks along $x y$ plane for periodic hexagonal arrays with different gaps and fixed diameter of $100 \mathrm{~nm}$ illuminated at their own resonance wavelength. It is interesting that with increasing the gap the electric field enhancement factor significantly increases and reaches a maximum value of about 2650 at a particular gap of $300 \mathrm{~nm}$. Previously, such strong enhancements were only obtained with nano-gap structures (via short-range dipoledipole interaction) but not easily seen in patterns with large intra-particle distance. Here, the interesting result suggests that a special long-range interaction plays an important role, which is very complicated and needs detailed research in near future.

\section{Conclusion}

In this study, we have presented the influence of diameter and the gap between nanodisks on SPR spectra and electric field enhancement factor for periodic hexagonal lattice arrays of silver nanodisks. An increase of extinction spectra, significant peak red-shift, and a broader plasmon resonance occur with increasing the diameter due to phase retardation of single large nanodisk and the near field coupling between nanodisks. FDTD calculations also show that with increasing the gap, electric field enhancement factor could significantly increase and reach a maximum value due to special long-range interaction. This study is useful for optical modulation applied in sensing and data storage, and solar cell, and is also important to the understanding plasmon 

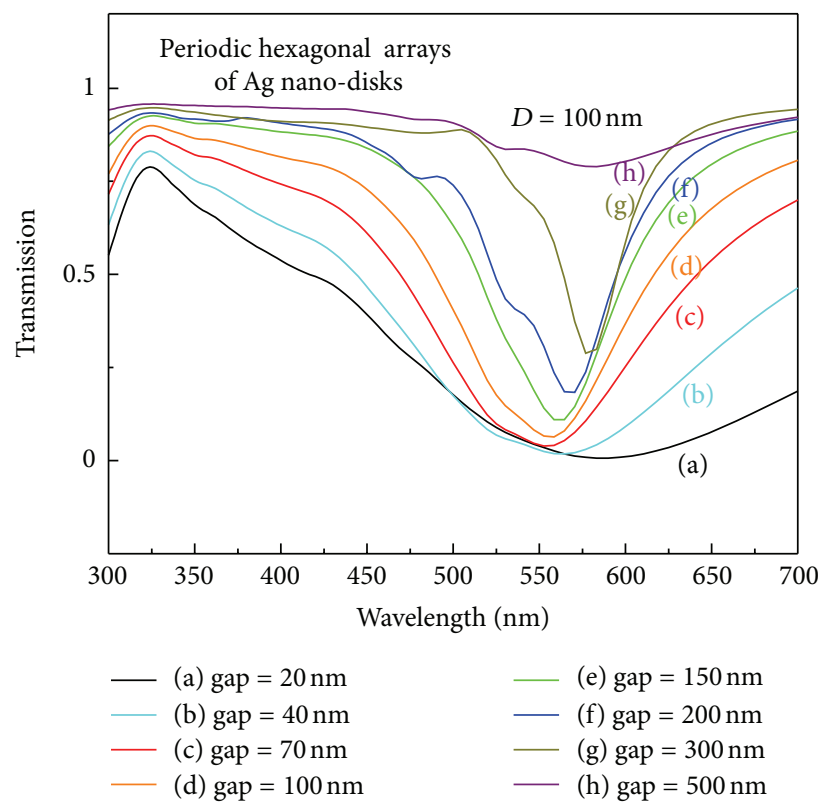

(a)

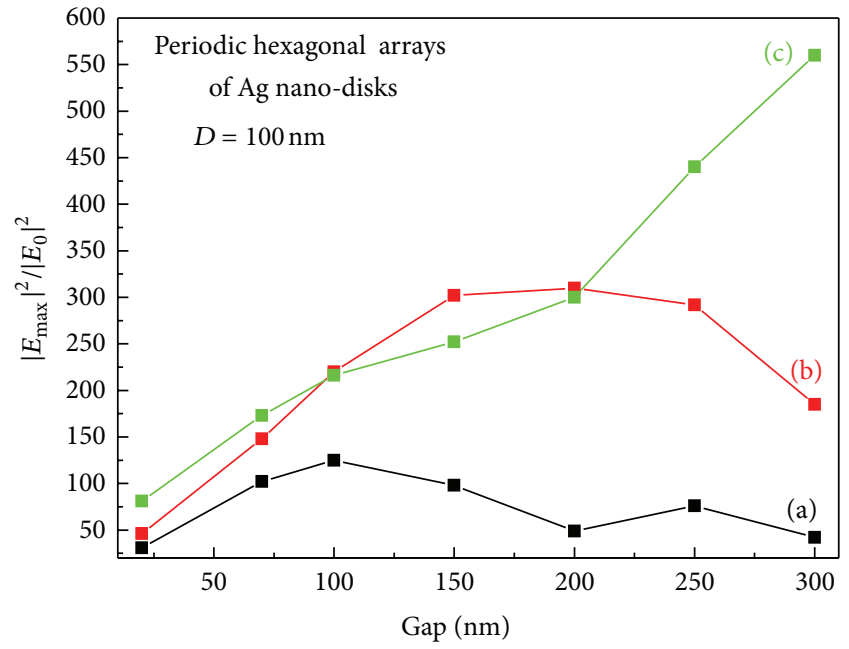

(a) $\lambda=488 \mathrm{~nm}$

(b) $\lambda=532 \mathrm{~nm}$

(c) $\lambda=632.8 \mathrm{~nm}$

(b)

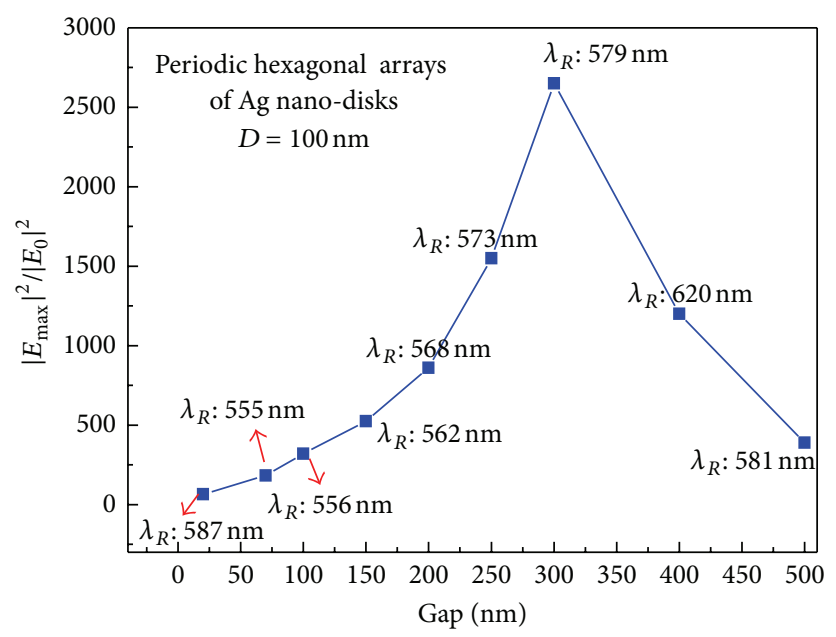

(c)

FIGURE 4: (a) The transmission spectra of periodic arrays with different gaps. (b) $E$-field enhancement factor on the top surface of nanodisks along $x y$ plane for periodic hexagonal arrays with different gaps illuminated at three laser wavelengths $(488 \mathrm{~nm}, 532 \mathrm{~nm}, 632.8 \mathrm{~nm})$. (c) $E$ field enhancement factor at the top of nanodisks along $x y$ plane for periodic hexagonal arrays with different gaps illuminated at their own resonance wavelengths. The diameter of the nanodisks is fixed at $100 \mathrm{~nm}$.

about near field coupling and long range interaction between nanoparticles.

\section{Acknowledgment}

This work was supported by the National Natural Science Foundation of China (Grant no. 50701002, Grant no. 51001078, Grant no. 11204317, and Grant no. 11274173).

\section{References}

[1] U. Kreibig and M. Vollmer, Optical Properties of Metal Clusters, Springer, New York, NY, USA, 1995.
[2] J. Hu, L. Chen, Z. Lian et al., "Deep-ultraviolet-blue-light surface plasmon resonance of $\mathrm{Al}$ and $\mathrm{Al}_{\text {core }} / \mathrm{Al}_{2} \mathrm{O}_{3 \text { shell }}$ in spherical and cylindrical nanostructures," Journal of Physical Chemistry C, vol. 116, no. 29, pp. 15584-15590, 2012.

[3] Y. Li, N. Koshizaki, H. Wang, and Y. Shimizu, "Untraditional approach to complex hierarchical periodic arrays with trinary stepwise architectures of micro-, submicro-, and nanosized structures based on binary colloidal crystals and their fine structure enhanced properties," ACS Nano, vol. 5, no. 12, pp. 9403-9412, 2011.

[4] J. Hu, L. Wang, W. Cai et al., "Smart and reversible surface plasmon resonance responses to various atmospheres for silver nanoparticles loaded in mesoporous $\mathrm{SiO}_{2}$," Journal of Physical Chemistry C, vol. 113, no. 44, pp. 19039-19045, 2009. 
[5] J. Hu, P. Liu, and L. Chen, "Comparison of surface plasmon resonance responses to dry/wet air for $\mathrm{Ag}, \mathrm{Cu}$, and $\mathrm{Au} / \mathrm{SiO}_{2}$," Applied Optics, vol. 51, no. 9, pp. 1357-1360, 2012.

[6] N. A. Cinel, S. Bütün, and E. Özbay, "Electron Beam Lithography designed silver nanodisks used as label free nanobiosensors based on Localized Surface Plasmon Resonance," Optics Express, vol. 20, no. 3, pp. 2587-2597, 2012.

[7] Y. Nishijima, L. Rosa, and S. Juodkazis, "Surface plasmon resonances in periodic and random patterns of gold nano-disks for broadband light harvesting," Optics Express, vol. 20, no. 10, pp. 11466-11477, 2012.

[8] G. Liu, Y. Li, G. Duan, J. Wang, C. Liang, and W. Cai, "Tunable surface plasmon resonance and strong SERS performances of Au opening-nanoshell ordered arrays," ACS Applied Materials and Interfaces, vol. 4, no. 1, pp. 1-5, 2012.

[9] G. Duan, F. Lv, W. Cai, Y. Luo, Y. Li, and G. Liu, "General synthesis of $2 \mathrm{D}$ ordered hollow sphere arrays based on nonshadow deposition dominated colloidal lithography," Langmuir, vol. 26, no. 9, pp. 6295-6302, 2010.

[10] Z. F. Dai, Y. Li, G.T. Duan, L.C. Jia, and W.P. Cai, "Phase diagram, design of monolayer binary colloidal crystals, and their fabrication based on ethanol-assisted self-assembly at the air/water interface," ACS Nano, vol. 6, no. 8, pp. 6706-6716, 2012.

[11] S. Yang, B. Cao, L. Kong, and Z. Wang, "Template-directed dewetting of a gold membrane to fabricate highly SERS-active substrates," Journal of Materials Chemistry, vol. 21, no. 36, pp. 14031-14035, 2011.

[12] E. D. Palik, Handbook of Optical Constants of Solids, Academic, New York, NY, USA, 1985.

[13] B. Lamprecht, G. Schider, R. T. Lechner et al., "Metal nanoparticle gratings: influence of dipolar particle interaction on the plasmon resonance," Physical Review Letters, vol. 84, no. 20, pp. 4721-4724, 2000.

[14] W. Khunsin, B. Brian, J. Dorfmüller et al., "Long-distance indirect excitation of nanoplasmonic resonances," Nano Letters, vol. 11, no. 7, pp. 2765-2769, 2012. 

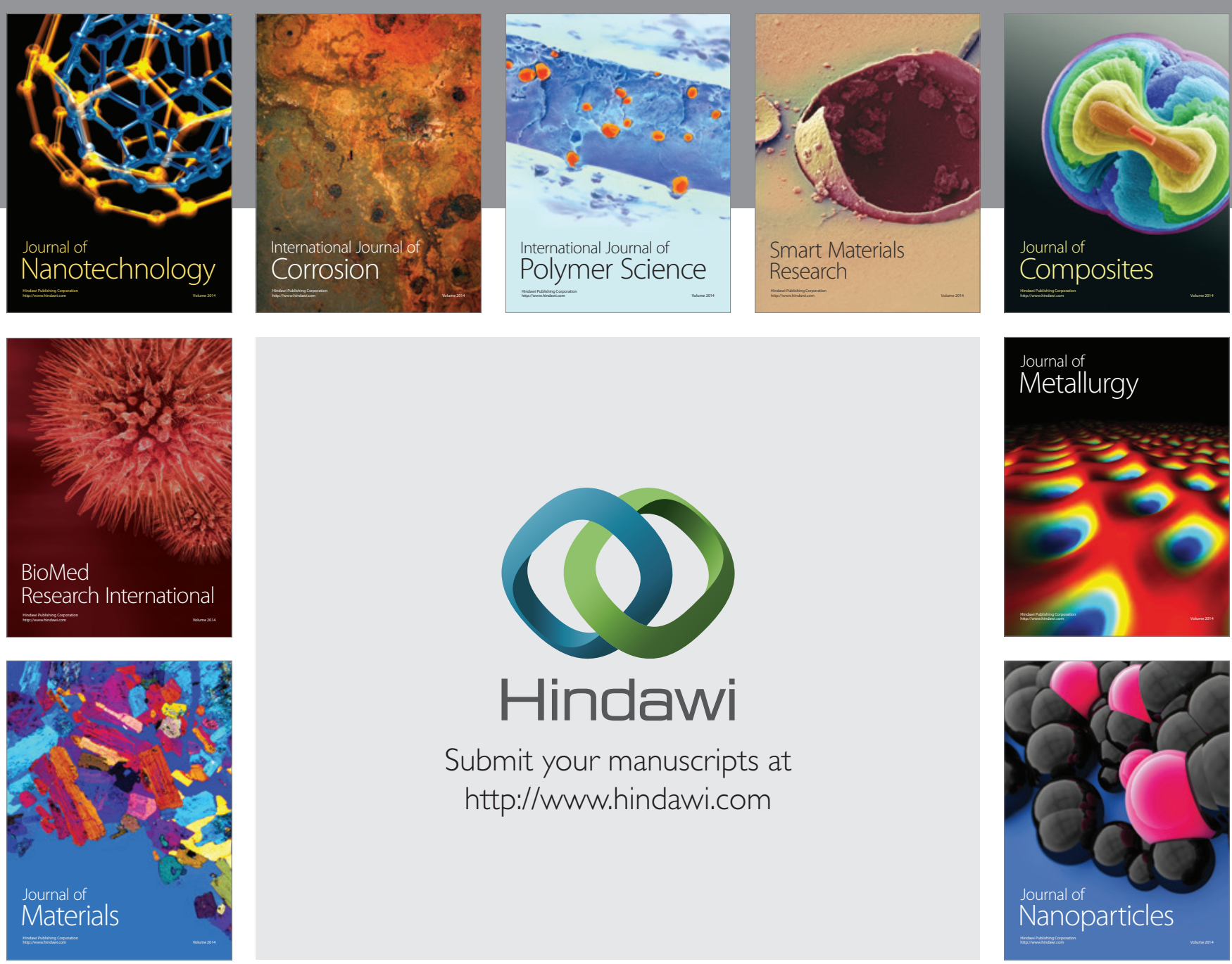

Submit your manuscripts at http://www.hindawi.com
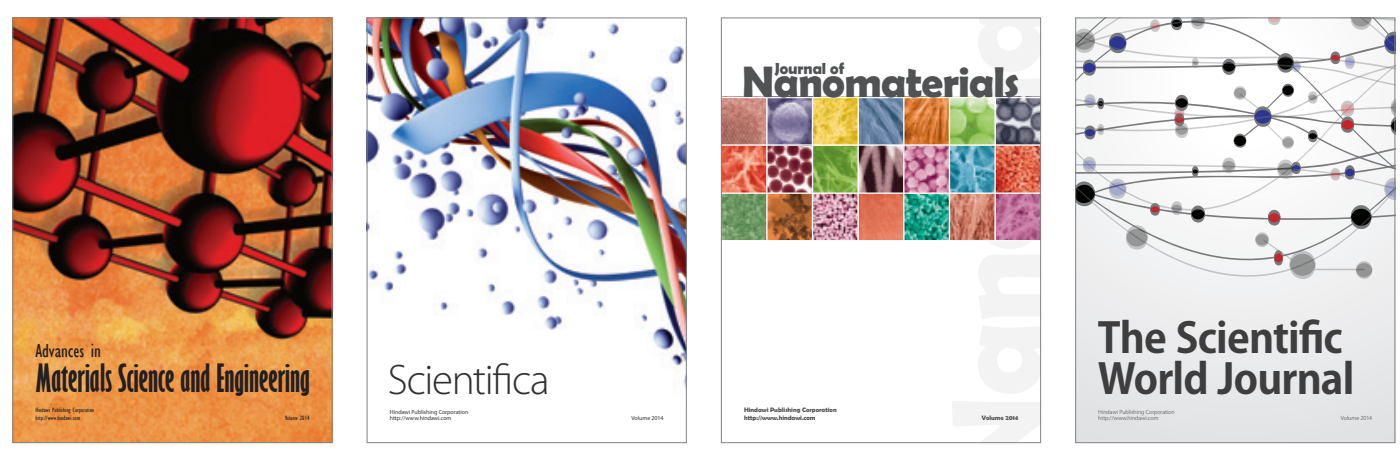

\section{The Scientific World Journal}
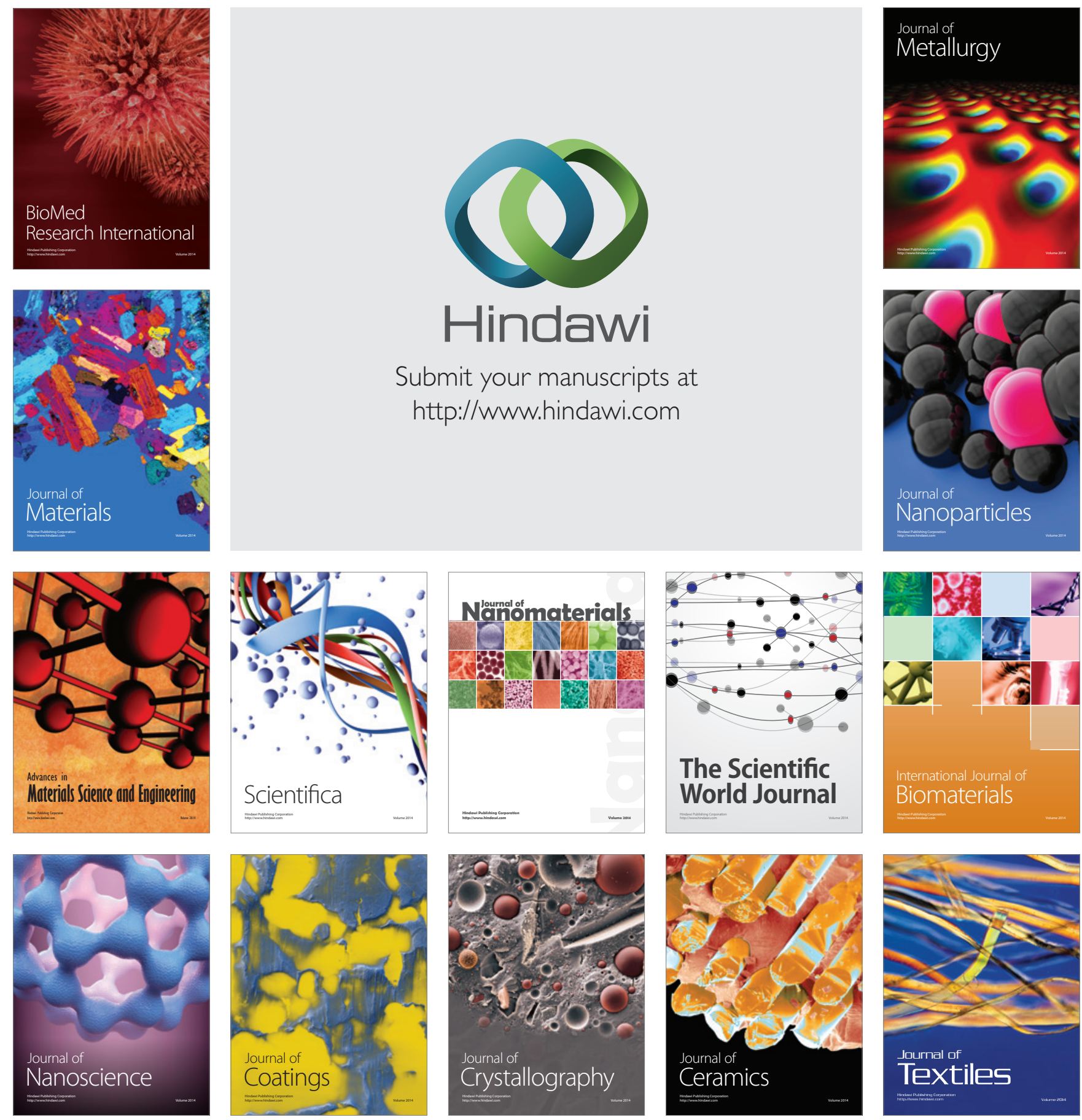